Marianna Csörnyei, Eötvös University, Department of Analysis, Budapest, Rákóczi út 5, H-1088, Hungary. e-mail: csornyei@cs.elte.hu

\title{
A NOTE ON STRONGLY INVISIBLE SETS
}

\author{
Abstract \\ Assuming the continuum hypothesis, there exists a strongly invisible \\ set which is visible from a visible set.
}

A set $A \subset \mathbb{R}^{2}$ is called invisible, if its orthogonal projection is of measure 0 in almost every direction; $A$ is visible, if it is not invisible. We say that $A$ is invisible from a point, if almost all lines through that point do not hit the set, except possibly for this point itself.

P. Mattila conjectured that the set of points from which an invisible set is visible is invisible. In [2] it is proved that this conjecture is false; there exists a visible set from which an invisible set is visible.

G. Petruska raised the question whether there exists a counterexample if instead of invisible sets we consider strongly invisible sets: a set $A \subset \mathbb{R}^{2}$ is called strongly invisible, if its orthogonal projection has strong measure 0 in almost every direction (recall that a set $X \subset \mathbb{R}$ has strong measure 0 , if for every positive sequence $\varepsilon_{1}, \varepsilon_{2}, \ldots$ there exists a sequence of intervals $I_{1}, I_{2}, \ldots$ for which $X \subset \bigcup_{n} I_{n}$ and $\left.\left|I_{n}\right|=\varepsilon_{n}\right)$.

Borel conjectured that all the sets of strong measure 0 are countable (see [1]). This conjecture is now known to be independent: assuming continuum hypothesis it is easy to construct a set of strong measure 0 whose cardinality is continuum. R. Laver proved in [3] that Borel's conjecture is consistent (the model necessarily satisfies the negation of the continuum hypothesis).

It is immediate to see that, if the projection of a set is countable in two different directions then the set itself is countable, and a countable set cannot be visible from any point. However, we show that assuming continuum hypothesis there exists a strongly invisible set which is visible from a visible set.

We prove a bit more general result:

\footnotetext{
Key Words: invisible set, strong measure zero

Mathematical Reviews subject classification: 28A05, 04A30

Received by the editors January 22, 1999

* Research supported by the Hungarian National Foundation for Scientific Research Grant No. F029768, T019476 and FKFP Grant 0189/1997.
} 
Theorem 1. Assuming continuum hypothesis, for every subset of directions $D$ there exist $K, L \subset \mathbb{R}^{2}$ such that

(A) given any direction in $D$, the projection of $K$ (in that direction) is the whole line;

(B) the projection of $K$ in all the other directions has strong measure 0;

(C) the projection of $L$ in every direction has strong measure 0;

(D) for every point $P \in K$ the set of the directions of lines connecting $P$ and $L$ contains the complement of $D$.

Now, if both $D$ and its complement is of positive measure then $K$ is visible, $L$ is strongly invisible, and $L$ is visible from all the points of $K$.

The proof of the theorem is based on the following lemma:

Lemma 1. Let $E=\left\{E_{\alpha}: \alpha<\omega_{1}\right\}$ be a set of lines and let $H=\left\{H_{\alpha}: \alpha<\right.$ $\left.\omega_{1}\right\}$ be a set of open dense planar sets for which $E_{\alpha} \cap H_{\beta}$ is an open dense set on $E_{\alpha}$ for every $\alpha, \beta<\omega_{1}$. Then there exists a set $X \subset \mathbb{R}^{2}$ for which $\left|X \cap E_{\alpha}\right| \geq 2$ and $\left|X \backslash H_{\alpha}\right| \leq \aleph_{0}$ for every $\alpha<\omega_{1}$.

Proof. Let $A=\left\{A_{\alpha}: \alpha<\omega_{1}\right\}$ be an enumeration of the set $E \cup H$. Then, for every $\alpha$ there exists a $\beta$ for which $E_{\alpha}=A_{\beta}$. Now,

$$
\bigcap_{\gamma<\beta, A_{\gamma} \in H} A_{\gamma}
$$

is a countable intersection of elements of $H$, all of them intersects $E_{\alpha}$ in an open dense set, thus applying Baire category theorem we have two different points $x_{\alpha}, y_{\alpha} \in E_{\alpha} \cap\left(\bigcap_{\gamma<\beta, A_{\gamma} \in H} A_{\gamma}\right)$. It is easy to see that the set $X=$ $\left\{x_{\alpha}, y_{\alpha}: \alpha<\omega_{1}\right\}$ satisfies the requirement $\left|X \cap E_{\alpha}\right| \geq 2$ for every $\alpha$, and since for every $\beta$ the points $x_{\alpha}, y_{\alpha}$ belong to $H_{\beta}$ for $\alpha$ large enough the set $X \backslash H_{\beta}$ has at most countable elements, as required.

Proof of The theorem. For every sequence $\underline{\varepsilon}=\left(\varepsilon_{1}, \varepsilon_{2}, \ldots\right)\left(\varepsilon_{j}>0\right)$ and for every direction $\theta \notin D$ we choose a set $G_{\underline{\varepsilon}, \theta}$ consisting of parallel lines for which

(i) the direction of the parallel lines is $\theta$;

(ii) there exists a dense open set of real intervals $I_{n}$ such that $\left|I_{n}\right|=\varepsilon_{n}$ for every $n$ and the projection of $G_{\underline{\varepsilon}, \theta}$ in direction $\theta$ is $\bigcup_{n} I_{n}$. 
Let $E^{\prime}$ be the set of the lines whose direction belongs to $D$, and let $H^{\prime}$ be the set of all the sets $G_{\underline{\varepsilon}, \theta}$. Since the directions of the parallel lines in $G_{\underline{\varepsilon}, \theta}$ does not belong to $D, G_{\varepsilon, \theta}$ intersects all the lines of direction in $D$ in a dense open set. Assuming continuum hypothesis we can apply our lemma for $E=E^{\prime}$ and $H=H^{\prime}$. The result is a set $X^{\prime}$ which intersects all the lines of direction in $D$ and whose projection in the other directions can be covered by a sequence of intervals $I_{n}$ of arbitrarily small length except countable many points. This implies immediately that $K=X^{\prime}$ satisfies conditions $(A)$ and $(B)$.

On the other hand, let $E^{\prime \prime}$ be the set of lines through the points of $K$ in directions not belonging to $D$, and for every direction $\theta$ and sequence $\underline{\varepsilon}$ we choose a set $G_{\underline{\varepsilon}, \theta}$ consisting of parallel lines for which (i), (ii) and applying (B) also

(iii) if $\theta \notin D$ then $K \subset G_{\underline{\varepsilon}, \theta}$

holds. Let $H^{\prime \prime}$ be the set of these sets $G_{\varepsilon, \theta}$. Again $E^{\prime \prime}$ and $H^{\prime \prime}$ satisfy the conditions of the lemma, we get a set $X^{\prime \prime}$ which intersects all the lines through the points of $K$ of direction not belonging to $D$ in at least one more point, and whose projections can be covered by a sequence of intervals of arbitrarily small length. Thus $L=X^{\prime \prime}$ satisfies conditions $(C)$ and $(D)$.

\section{References}

[1] E. Borel, Sur la classification des ensembles de mesure nulle, Bull. Math. France, 47(1919), 97-125.

[2] M. Csörnyei, On the visibility of invisible sets, to appear in Academiæ Scientiarum Fennicæ

[3] R. Laver, On the consistency of Borel's conjecture, Acta. Math., 137(1976), 151-169. 
